\title{
Improved molecular characterization of the Klebsiella oxytoca complex reveals the prevalence of the kleboxymycin biosynthetic gene cluster
}

Preetha Shibu' †, †, Frazer McCuaig²†, Anne L. McCartney ${ }^{3}$, Magdalena Kujawska ${ }^{4}$, Lindsay J. Hall4,5 and Lesley Hoyles ${ }^{2,6, *}$

\begin{abstract}
As part of the ongoing studies with clinically relevant Klebsiella spp., we characterized the genomes of three clinical GES-5-positive ST138 strains originally identified as Klebsiella oxytoca. bla $a_{\text {oxy }}$ gene, average nucleotide identity and phylogenetic analyses showed the strains to be Klebsiella michiganensis. Affiliation of the strains to ST138 led us to demonstrate that the current multi-locus sequence typing scheme for $K$. oxytoca can be used to distinguish members of this genetically diverse complex of bacteria. The strains encoded the kleboxymycin biosynthetic gene cluster (BGC), previously only found in K. oxytoca strains and one strain of Klebsiella grimontii. The finding of this BGC, associated with antibiotic-associated haemorrhagic colitis, in K. michiganensis led us to carry out a wide-ranging study to determine the prevalence of this BGC in Klebsiella spp. Of 7170 publicly available Klebsiella genome sequences screened, 88 encoded the kleboxymycin BGC. All BGC-positive strains belonged to the K. oxytoca complex, with strains of four (K. oxytoca, K. pasteurii, K. grimontii, K. michiganensis) of the six species of complex found to encode the complete BGC. In addition to being found in $K$. grimontii strains isolated from preterm infants, the BGC was found in K. oxytoca and K. michiganensis metagenome-assembled genomes recovered from neonates. Detection of the kleboxymycin BGC across the K. oxytoca complex may be of clinical relevance and this cluster should be included in databases characterizing virulence factors, in addition to those characterizing BGCs.
\end{abstract}

\section{DATA SUMMARY}

Draft genome sequences for PS_Koxy1,PS_Koxy2 and PS_Koxy4 have been deposited with links to BioProject accession number PRJNA562720 and under accession numbers VTQC00000000, VTQB00000000 and VTQA00000000, respectively. Supplementary data and material associated with this article are available from figshare at https://figshare.com/projects/Kleboxymycin_ biosynthetic_gene_cluster/81059.

\section{INTRODUCTION}

Members of the Klebsiella oxytoca complex encode a chromosomal $\beta$-lactamase gene $\left(b l a_{\mathrm{OXY}}\right)[1]$. Differences in the sequence of this gene allowed the establishment of phylogroups (Ko), which correspond to species: K. michiganensis (Ko1, with Ko5 representing a sub-lineage), K. oxytoca (Ko2), K. spallanzanii (Ko3), K. pasteurii (Ko4), K. grimontii (Ko6) and K. huaxiensis (Ko8). Ko7 has been described on the basis of a single isolate [1]. Individual gene ( $r p o B, g y r A, r r s)$ sequences can be used to differentiate species of the complex [2], as can genome-based average nucleotide identity (ANI) and phylogenomic analyses $[1,3]$. All members of the K. oxytoca complex can be differentiated by MALDI-TOF [1], but reference databases currently in routine clinical use lack reference spectra of the different species to allow identification beyond $K$. oxytoca.

Received 04 August 2020; Accepted 25 April 2021; Published 18 June 2021

Author affiliations: 'Life Sciences, University of Westminster, UK; ${ }^{2}$ Department of Biosciences, Nottingham Trent University, UK; ${ }^{3}$ Department of Food and Nutritional Sciences, University of Reading, UK; ${ }^{4}$ Gut Microbes \& Health, Quadram Institute Bioscience, Norwich Research Park, Norwich, UK; ${ }^{5} \mathrm{Chair}$ of Intestinal Microbiome, ZIEL - Institute for Food \& Health, Technical University of Munich, Freising, Germany; ${ }^{6}$ Department of Surgery and Cancer, Imperial College London, UK.

*Correspondence: Lesley Hoyles, lesley.hoyles@ntu.ac.uk

Keywords: tilimycin; tillivaline; Klebsiella michiganensis; antibiotic-associated haemorrhagic colitis; necrotizing enterocolitis.

Abbreviations: AAHC, antibiotic-associated haemorrhagic colitis; AMR, antimicrobial resistance; BGC, biosynthetic gene cluster; MAG,

metagenome-assembled genome; MLST, multi-locus sequencing typing; MSA, multiple-sequence alignment; NEC, necrotizing enterocolitis; PBD,

pyrrolobenzodiazepine; rMLST, ribosomal MLST; TM, tilimycin; TV, tillivaline; VFDB, Virulence Factors of Pathogenic Bacteria Database.

$\ddagger$ Present address: Berkshire and Surrey Pathology Services, Frimley Health NHS Trust, Wexham Park Hospital, Slough, UK.

†These authors contributed equally to this work

Data statement: All supporting data, code and protocols have been provided within the article or through supplementary data files. Five supplementary tables and five supplementary figures are available with the online version of this article.

$000592 \odot 2021$ The Authors 
Recent work has demonstrated genomic characterization of $K$. oxytoca strains is inadequate, with large numbers of genomes deposited in public databases erroneously assigned to K. oxytoca instead of K. michiganensis or K. grimontii [3-6]. Consequently, K. michiganensis and K. grimontii are clinically relevant but under-reported in the literature [3,7]. Given that the $b l a_{\mathrm{OXY}}$ gene has diversified in parallel to housekeeping genes in the K. oxytoca complex, it is likely that the K. oxytoca multi-locus sequence typing (MLST) scheme [8] can be used to distinguish members of this genetically diverse group of bacteria.

Little is known about the antibiotic-resistance and virulence genes encoded by $K$. oxytoca and related species. In the course of ongoing Klebsiella-phage work, with three GES-5-positive ST138 strains originally described as $K$. oxytoca $[9,10]$, we sought to determine whether widely recognized virulence factors such as enterobactin, yersiniabactin and salmochelin are encoded in the strains' genomes, and the kleboxymycin biosynthetic gene cluster (BGC), as this was until recently a little-studied BGC implicated in non-Clostridioides difficile antibiotic-associated haemorrhagic colitis (AAHC) [11-14]. AAHC is caused by the overgrowth of cytotoxin-producing K. oxytoca secondary to use of antibiotics such as penicillin or amoxicillin, resulting in the presence of diffuse mucosal oedema and haemorrhagic erosions $[15,16]$. This type of colitis is distinct from the more common form of antibioticassociated diarrhoea caused by toxin-producing Clostridiodes difficile, which usually gives rise to watery diarrhoea resulting in mild to moderate disease.

Gene-based and genomic analyses of our ST138 isolates showed they were $K$. michiganensis, not $K$. oxytoca, and that along with common virulence genes they encoded the kleboxymycin BGC. Our findings led us to (1) determine whether the K. oxytoca MLST scheme could be used to differentiate members of the K. oxytoca complex, and (2) investigate the distribution of the kleboxymycin BGC in a range of Klebsiella and related species.

\section{METHODS}

\section{Clinical isolates}

Strains PS_Koxy1 (isolated December 2014; cardiothoracic/ intensive care unit), PS_Koxy2 (isolated August 2015; haematology unit) and PS_Koxy4 (isolated September 2015; haematology unit) had been recovered from a throat swab, urine and rectal swab, respectively, obtained from three different adults. The strains were from the study of Eades et al. [9], described in further detail by Ellington et al. [10] (PS_Koxy1, patient X; PS_Koxy2, patient A; PS_Koxy4, patient B; Frances Davies, personal communication). Full details of methods associated with the phenotypic and genotypic characterization of the clinical isolates can be found in Supplementary Material (available in the online version of this article).

\section{Impact Statement}

Members of the Klebsiella oxytoca complex are difficult to tell apart using phenotypic and chemotaxonomic methods. Consequently, many genomes deposited in public databases are misclassified as K. oxytoca. Here we demonstrate that the current multi-locus sequence typing (MLST) system for the complex can be used to accurately distinguish many strains, which will be of use to clinical laboratories in resource-limited settings which rely on the MLST scheme for typing and epidemiological tracking of isolates. In addition, extended analyses of the genomes of Klebsiella spp. have revealed the kleboxymycin biosynthetic gene cluster (BGC) is restricted to species of the Klebsiella oxytoca complex (K. oxytoca, K. michiganensis, K. pasteurii and K. grimontii). Species- and/ or gene-specific differences in the cluster's sequences may be relevant to virulence of $K$. oxytoca and related species. The finding of the kleboxymycin BGC in the preterm infant gut microbiota may have implications for disease presentation in a subset of neonates.

\section{ANI analysis of genome sequences}

All annotated non-redundant Klebsiella genome assemblies available in the NCBI Genome database on 2 September 2019 ( $n=7170$; Table S1) were downloaded [17]. ANI of genomes with their closest relatives and type strains of species was assessed using FastANI [18], which uses Mashmap as its MinHash-based alignment-free sequence mapping engine to provide ANI values for both complete and draft-quality genomes that are related by $80-100 \%$ ANI.

\section{MLST analyses}

Allele sequences ( $n=442$ representing seven housekeeping genes - gapA, infB, $m d h$, pgi, phoE, rpoB, ton B - contributing to 354 different MLST sequence types; correct as of 19 March 2021) for the K. oxytoca MLST scheme [8] were used to determine the MLST profiles of all K. oxytoca complex genomes included in this study (Table S1). The allele sequences were used to create BLASTN databases against which the assemblies of all genomes included in this study were searched. Sequences with exact hits to one allele of each housekeeping gene were retained, allowing us to identify the sequence types of the genomes included in this study (Table S2). For those genomes that returned hits to alleles across all seven housekeeping genes, a phylogenetic tree (neighbour joining, Jukes Cantor) was generated in Geneious Prime v2019.2.1 using the aligned (CLUSTAL W) concatenated ( $g a p A-i n f B-m d h-p g i-p h o E-r p o B-\operatorname{ton} B)$ nucleotide sequences of their housekeeping genes and those of each sequence type used in the K. oxytoca MLST scheme [8]. Support for clustering of nodes in the tree was determined by bootstrap analysis (1000 replications). 


\section{Characterization of the kleboxymycin BGC in genomes}

The annotated reference sequence of the kleboxymycin BGC was downloaded from GenBank (accession number MF401554 [11]) and used as a BLASTP database for searches with the protein sequences encoded within the genomes of PS_Koxy1, PS_Koxy2 and PS_Koxy4. Initially, Geneious Prime v2019.2.1 was used to identify regions of the three draft genomes encoding the complete BGC, and to align them to MF401554.

The protein sequences of the annotated assemblies were searched for the kleboxymycin BGC using the reference sequence and BLASTP v2.9.0+, and the resulting hits were filtered based on $>70 \%$ identity and $>70 \%$ coverage to identify isolates potentially carrying genes from the BGC. $K$. grimontii $(n=3)$ and $K$. michiganensis $(n=2)$ and $K$. oxytoca-related metagenome-assembled genomes (MAGs) ( $n=25)$ from Chen et al. [3] were also subject to BLASTP searches. Genomes that encoded the full BGC (i.e. all 12 BGC genes on a contiguous stretch of DNA) were identified from the BLAST results. The protein sequences encoded in the BGC were extracted from the annotated assemblies using samtools v1.9 faidx [19] and concatenated into a single sequence (the sequence data are available as supplementary material from figshare). These concatenated sequences were used to produce a multiple-sequence alignment (MSA) in Clustal Omega v1.2.4, along with the BGC sequences of the three $K$. michiganensis clinical isolates, the reference sequence [11], a recently described K. grimontii sequence [20] and a homologous sequence found in Pectobacterium brasiliense BZA12 (to be used as an outgroup in later phylogenetic analyses; identified as encoding the complete kleboxymycin BGC through NCBI BLASTP). Phylogenetic analyses were carried out on the MSA using the R package Phangorn v2.5.5 [21], producing a maximum-likelihood tree, which was visualised and rooted (on P. brasiliense BZA12) using the Interactive Tree of Life (iTOL v5.5) [22]. To examine variation at the individual protein level, further within-species MSAs were produced for each of the 12 protein sequences in the BGC. Each of these alignments was used as the basis for a consensus sequence, produced using EMBOSS Cons v6.6.0.0, representing each of the four species carrying the BGC. An MSA and per cent identity matrix were then generated for each protein between the consensus sequences of K. oxytoca, K. grimontii, K. michiganensis and K. pasteurii, along with the reference sequence [11].

The species affiliations of the genomes encoding the full kleboxymycin BGC were determined using FastANI v1.2 [18] against genomes of type strains of the K. oxytoca and $K$. pneumoniae complexes $[1,23]$ and $K$. aerogenes ATCC $13048^{\mathrm{T}}$ (assembly accession number GCA_003417445), with PhyloPhlAn 0.99 used to conduct a phylogenetic analysis to confirm species affiliations. PhyloPhlAn identifies hundreds of conserved (core) proteins from a given genomic dataset and uses them to build a complete high-resolution phylogeny.

\section{RESULTS \\ Characterization of the clinical isolates}

Although initial phenotypic tests (Supplementary Material) and genomic analyses $[9,10]$ identified PS_Koxy1, PS_Koxy2 and PS_Koxy4 as K. oxytoca, analyses of the isolates' proteomes showed them to be K. michiganensis ST138 (phylogroup Ko1, bla $\left.a_{\mathrm{OXY1-8}}\right)($ Fig. S1). Full details of phenotypic characterization and genome sequencing of the clinical isolates can be found in Supplementary Material. PS_Koxy1, PS_Koxy2 and PS_Koxy4 all shared $98.81,98.71$ and $98.71 \%$ ANI, respectively, with the type strain of K. michiganensis (W14 ${ }^{\mathrm{T}}$, GCA_901556995), and 99.98-100.00\% ANI with each other. Based on current recommendations, ANI of $95-96 \%$ and above with the genome of the type strain is indicative of species affiliation [24]. Inclusion of the genomes with representatives of all six species of the K. oxytoca complex in a phylogenetic analysis confirmed the affiliation of PS_Koxy1, PS_Koxy2 and PS_Koxy4 with K. michiganensis (Fig. S2).

\section{Assigning MLST sequence types to species}

While annotations for genomes are improving, we have previously noted and continue to notice issues with identities attributed to K. oxytoca genomes in public repositories [3]. Consequently, the identity of all genomes included in this work was first confirmed by ANI analysis (Table S1), with $b l a_{\mathrm{OXY}}$ gene and phylogenetic analyses supporting our findings (Supplementary Material). Of the $178 \mathrm{~K}$. oxytoca complex genomes identified, many had been misassigned in GenBank: seven genomes were listed as K. grimontii, 106 as K. oxytoca, 51 as K. michiganensis, 13 as Klebsiella sp. and one as K. pneumoniae. Our analyses of the 178 genomes showed the dataset actually represented $K$. michiganensis $(n=76), K$. oxytoca $(n=66), K$. grimontii $(n=24), K$. pasteurii $(n=6), K$. huaxiensis $(n=5)$ and K. spallanzanii $(n=1)$.

The $K$. oxytoca MLST scheme uses sequence polymorphisms among seven housekeeping genes - gapA, infB, $m d h$, pgi, phoE, rpoB, ton $B$ - to generate sequence types for isolates. Currently, there are 442 allele sequences that contribute to 354 unique MLST sequence types. We first identified nucleotide sequences within the genomes with exact matches to nucleotide sequences within the allele reference dataset. One-hundred-and-twenty-nine genomes returned hits to known MLST profiles, and 10 isolates returned MLST profiles with no assigned sequence type (Table S2). Our clinical isolates returned the expected ST138 result.

Of the $66 \mathrm{~K}$. oxytoca genomes, 59 could be assigned to known sequence types (in order of abundance: ST2, ST176, ST199, ST36, ST19, ST30, ST53, ST101, ST18, ST31, ST34, ST48, ST58, ST59, ST141, ST145, ST153, ST181, ST221, ST222, ST257, ST258, ST287, ST323) and one (GCA_003937225) represented a novel sequence type. Of the $24 \mathrm{~K}$. grimontii genomes, 13 could be assigned to known sequence types (ST172, ST216, ST104, ST186, ST236, ST263, ST316, ST319, ST350), with four (GCA_002856195, GCA_900451335, GCA_008120915, GCA_004343645) representing unique novel sequence 


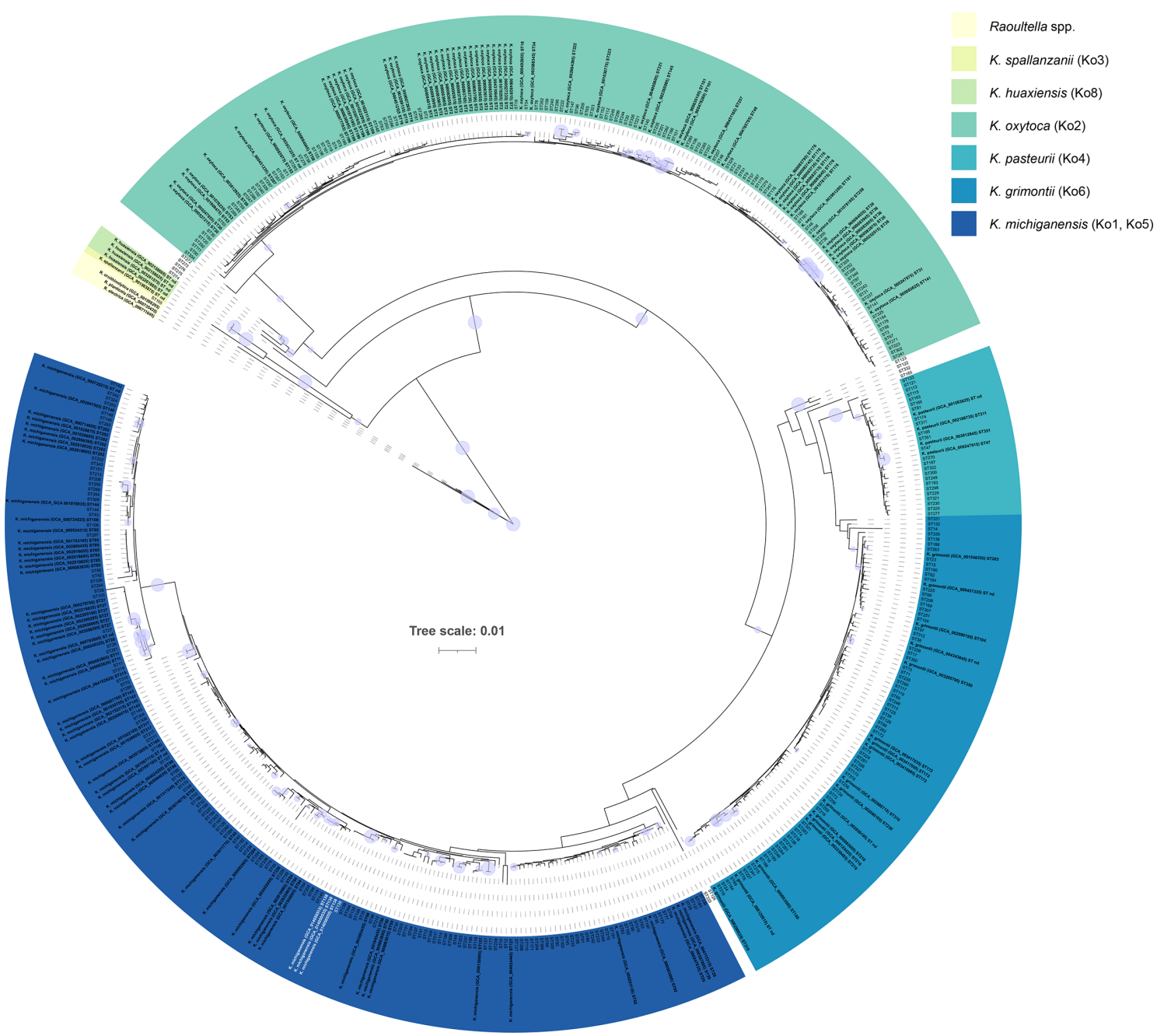

Fig. 1. Sequence types within the K. oxytoca MLST scheme can be used to distinguish members of the $K$. oxytoca complex. The three $K$. michiganensis clinical isolates (all ST138) characterized in this study are shown in white. The phylogenetic tree (neighbour joining, Jukes Cantor) was generated using concatenated nucleotide sequences of housekeeping genes (gapA-infB-mdh-pgi-phoE-rpoB-tonB) used in the K. oxytoca MLST scheme [8]. The purple circles represent bootstrap values $\geq 80 \%$ (based on 1000 replications); the larger the circle, the higher the bootstrap value. Scale bar, average number of nucleotide substitutions per position. The full list of MLST sequence types and their species affiliations are available in Table S2.

types. Of the six $K$. pasteurii genomes, three could be assigned to known sequence types (ST47, ST311, ST351) and one (GCA_901563825) represented a novel sequence type. Of the $79 \mathrm{~K}$. michiganensis genomes (including our three clinical isolates), 57 could be assigned to known sequence types (ST85, ST27, ST202, ST143, ST29, ST50, ST84, ST138, ST11, ST88, ST317, ST28, ST40, ST52, ST82, ST92, ST98, ST108, ST127, ST144, ST146, ST157, ST170, ST180, ST226, ST294, ST315), with four genomes (GCA_000783895, GCA_000735215, GCA_007097185, GCA_007097115) representing three novel sequence types. None of the K. huaxiensis or K. spallanzanii genomes returned hits to known alleles (Table S2), but the relevant individual housekeeping gene sequences are provided as Supplementary Files for use by other researchers.
For those genomes that encoded known or novel sequence types, we concatenated their housekeeping-gene sequences and used them to create a MSA with the concatenated sequences of each of the 354 recognized MLST sequence types. This MSA was used to create a phylogenetic tree, allowing us to visualize the relationships among species and sequence types (Fig. 1).

Of the 354 known MLST sequence types, 342 (96.6\%) were associated with specific members of the K. oxytoca complex (Table S2): 115 with K. oxytoca, 130 with K. michiganensis, 73 with $K$. grimontii and 24 with K. pasteurii. Eleven were associated with unspecified members of the $K$. oxytoca complex. ST105 was associated with Raoultella ornithinolytica, sharing 99.73\% sequence similarity type strain's MLST profile. K. 


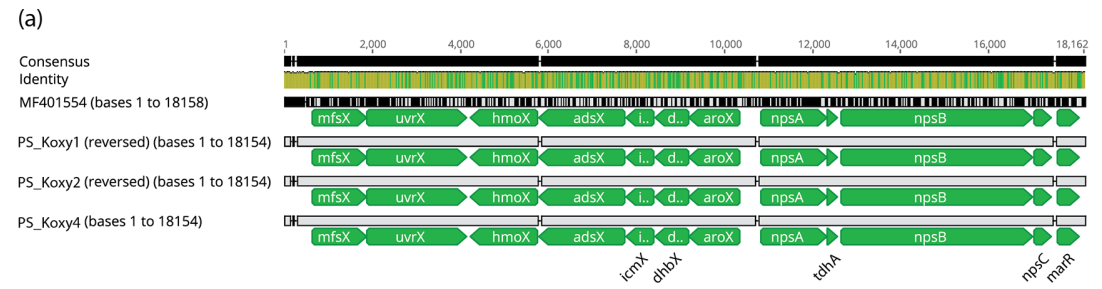

(b)

Fig. 2. Alignment of the kleboxymycin BGCs from the three clinical K. michiganensis strains with the complete cluster of K. oxytoca MH431 (GenBank accession number MF401554 [11]). (a) The image (alignment view) was generated via the progressiveMauve algorithm plugin of Geneious Prime v2019.2.1 (default settings, full alignment), with gene names for the three clinical isolates assigned manually. (b) Genes corresponding to Prokka-generated annotations. Consensus identity is the mean pairwise nucleotide identity over all pairs in the column: green, 100\% identity; greeny-brown, at least 30\% and under 100\% identity; red, below 30\% identity.

oxytoca-specific sequence types shared $98.64-100 \%$ sequence similarity, K. michiganensis-specific sequence types shared 96.62-100.00\% sequence similarity, K. grimontii-specific sequence types shared $98.20-100.00 \%$ sequence similarity, K. pasteurii-specific sequence types shared 99.00$100.00 \%$ sequence similarity and $K$. huaxiensis-specific sequence types shared $97.09-99.7 \%$ sequence similarity. A matrix of similarity values for the 504 sequences included in the analysis is available in Supplementary Material, along with the MSA alignment used to generate the phylogenetic tree shown in Fig. 1.

\section{Detection of the complete kleboxymycin BGC in clinical isolates}

It has long been known that $K$. oxytoca gut colonization is linked with AAHC [16]. Schneditz et al. [12] showed tillivaline (TV), a pyrrolobenzodiazepine (PBD) derivative produced by K. oxytoca, is one of the enterotoxins responsible for causing AAHC. This toxic product is encoded by the heterologous expression of the kleboxymycin (also known as tilimycin (TM) [14]) BGC comprising 12 genes [11]. Protein sequences of the reference sequence [11] were used to create a BLASTP database against which the proteins encoded in the genomes of PS_Koxy1, PS_Koxy2 and PS_Koxy4 were compared. The genomes of PS_Koxy1, PS_Koxy2 and PS_Koxy4 encoded a complete kleboxymycin BGC (Fig. 2). All genes in each of the genomes shared $>99 \%$ identity and $>99 \%$ query coverage with the genes of the reference sequence [12]: $m f_{s} X, 99.76 \%$ identity; $u v r X, 99.87 \%$; $h m o X, 99.80 \%$; ads $X, 99.85 \%$; icmX, 99.52\%; dhbX, 99.62\%; aroX, 99.74\%; npsA, 99.80\%; thdA, 98.68\%; npsB, 99.93\%; npsC, 98.47\%; marR, 99.39\%.

Our strains were K. michiganensis ST138, so we downloaded and assembled (from BioProject PRJEB30858) available raw sequence data from $19 \mathrm{~K}$. oxytoca ST138 strains described recently [10] and determined whether they were in fact $K$. michiganensis and encoded the kleboxymycin BGC. All strains were confirmed to be K. michiganensis on the basis of
ANI analysis, and encoded the complete kleboxymycin BGC (Fig. S4).

Schneditz et al. [12] reported $n p s A / n p s B$ were functionally conserved in six sequenced strains of $K$. oxytoca (Table 1 ), based on a BLASTP analysis. Full details of the analysis are unavailable, with only a brief mention of presence being determined based on BLASTP sequence identities $>90 \%$ with no indication of sequence coverage. All the genomes included in the study of Schneditz et al. [12] were compared with those of the type strain of $K$. oxytoca and related species to confirm their species affiliations (Table 1). While some strains were K. oxytoca, others belonged to K. michiganensis, $K$. pasteurii, K. grimontii and R. ornithinolytica. Using thresholds of $70 \%$ identity and $70 \%$ query coverage in our BLASTP analyses to reduce the potential for detecting false positives, we reanalysed the genomes included in the study of Schneditz et al. [12]. Our results agreed with those of Schneditz et al. [12] for all genomes, except we detected $n p s A / n p s B$ (and all other genes encoded in the kleboxymycin BGC) in K. grimontii SA2. K. oxytoca 10-5243, K. pasteurii 10-5250, K. oxytoca $11492-1, K$. oxytoca $10-5248$ and K. grimontii M5a1 also encoded the whole kleboxymycin BGC. All genes in all matches shared greater than $90 \%$ identity across greater than $99 \%$ query coverage. K. michiganensis $10-5242$, E718 and KCTC 1686 did not encode homologues associated with the kleboxymycin BGC. K. oxytoca 10-5245 encoded almostcomplete homologues of four genes [EHS96696.1 (marA) 98.79\% identity, 99.39\% coverage; EHS96697.1 (npsC) 95.38\% identity, 99.23\% coverage; (EHS96698.1 ( $m f s X)$ 96.68\% identity, 99.87\% coverage; EHS96699.1 (uvrX) 94.88\% identity, 99.76\% coverage] in contig JH603137.1.

\section{Detection of the kleboxymycin BGC in the faecal microbiota of preterm infants}

Our previous work had highlighted the preterm infant gut microbiota harbours a range of species belonging to the K. oxytoca complex [3]. BLASTP searches of the two K. michiganensis 
Table 1. Genomes included in analyses conducted by Schneditz et al. [12] with corrected species affiliations (originally reported as K. oxytoca)

\begin{tabular}{|c|c|c|c|c|}
\hline Assembly accession & Strain & Species & ANI with shown genome $e^{\star}$ & $n p s A / n p s B$ \\
\hline GCA_000247835.1 & $10-5242$ & K. michiganensis & 97.59\%, GCA_901556995.1 & - \\
\hline GCA_000247855.1 & $10-5243$ & K. oxytoca & 99.31\%, GCA_900977765.1 & + \\
\hline GCA_000247875.1 & $10-5245$ & K. oxytoca & 99.13\%,GCA_900977765.1 & - \\
\hline GCA_000247915.1 & $10-5250$ & K. pasteurii & 99.29\%, GCA_901563825.1 & + \\
\hline GCA_000252915.3 & $11492-1$ & K. oxytoca & 99.15\%, GCA_900977765.1 & + \\
\hline GCA_000276705.2 & E718 & K. michiganensis & 98.37\%, GCA_901556995.1 & - \\
\hline GCA_000427015.1 & SA2 & K. grimontii & 99.33\%, GCA_900200035.1 & + \\
\hline
\end{tabular}

*GCA_901556995.1=K. michiganensis W14 ${ }^{\top}$; GCA_900200035.1, K. grimontii 06D021'T; GCA_900977765.1=K. oxytoca ATCC 13182';

GCA_001598295.1=R. ornithinolytica NBRC 105727'; K. pasteurii SB3355' GCA_901563825.1.

(P049A W, GCA_008120305; P095L Y, GCA_008120085) and three K. grimontii (P038I, GCA_008120465; P043G P, GCA_008120425; P079F P, GCA_008120915) strains we previously characterized showed all three $K$. grimontii strains encoded the kleboxymycin BGC (Fig. S5). All BGC genes in their genomes shared $>98 \%$ identity and $>99 \%$ query coverage with the genes of the reference sequence [11]: $m f_{s} X, 100 \%$ identity; uvrX, 99.60-99.73\%; hmoX, 99.80\%; adsX, 99.69\%; icmX, 100\%; dhbX, 100\%; aroX, 99.94-99.74\%; npsA, 99.21-99.41\%; thd A, 98.68\%; npsB, 99.31-99.38\%; npsC, 99.23-100\%; marR, $100 \%$. The BGC was also detected in 8 out of 25 of the pretermassociated $K$. oxytoca complex MAGs (three $K$. oxytoca, five K. michiganensis) we described previously [3]. An MSA of the preterm-associated genomes' BGC against the reference sequence [11] suggested species-specific clustering of the sequences (Fig. S5).

\section{Prevalence of the kleboxymycin BGC in Klebsiella spp.}

Given the work detailed above had detected the kleboxymycin BGC in several different but closely related Klebsiella species and in a range of clinical and gut-associated isolates, and Hubbard et al. [20] recently detected the BGC in a strain of $K$. grimontii, we chose to increase the scope of our analysis to include 7170 publicly available assembled Klebsiella genomes (including our three clinical strains, and five isolates from preterm infants [3]) (Table S1).

As mentioned above, we have noted issues with identities attributed to Klebsiella genomes in public repositories [3], so the identity of all non- $K$. oxytoca complex genomes included in this work was first confirmed by ANI analysis (Table S1). The majority $(n=6245)$ of the additional genomes were $K$. pneumoniae, followed by $K$. variicola subsp. variicola $(n=241), K$. quasipneumoniae subsp. similipneumoniae $(n=184), K$. aerogenes $(n=168), K$. quasipneumoniae subsp. quasipneumoniae $(n=120), K$. variicola subsp. tropica $(n=19)$, ' $K$. quasivariicola' $(n=11)$ and $K$. africana $(n=1)$. Out of 7170 genomes, $110(1.5 \%)$ had one or more matches with the 12 genes encoded within the kleboxymycin BGC reference sequence, with all except two genomes (both $K$. pneumoniae) belonging to species of the $K$. oxytoca complex (Table S3). Ninety-six genomes - all belonging to the K. oxytoca complex - encoded at least 12 genes belonging to the BGC (Table S3), and were examined further.

One genome (GCA_002856195) encoding 12 BGC genes was found to encode two stretches of the same protein with the other cluster-associated genes non-contiguous, while one (GCA_004005605) encoded 13 BGC genes (one gene duplicated) in a non-contiguous arrangement. Fifty-five out of $66(83.3 \%)$ K. oxytoca genomes encoded the entire kleboxymycin BGC, as did 19 out of $24(79.2 \%)$ K. grimontii, 9 out of 79 (11.4\%) K. michiganensis and five out of six (83.3\%) K. pasteurii genomes (Fig. 3a). Phylogenetic analysis (Fig. 3b) confirmed findings from ANI analyses (Table S1) that showed all genomes belonged to species of the K. oxytoca complex. The 88 genomes confirmed to encode the complete kleboxymycin BGC included the type strain of K. grimontii. The BGC cluster sequences grouped according to species, and the reference sequence [11] clustered with $K$. grimontii sequences and was closely related to the type strain of that species (Fig. 3c).

Species-specific consensus sequences were generated for all genes within the kleboxymycin BGC and are available as supplementary material from figshare. Similarity values for 
(a)

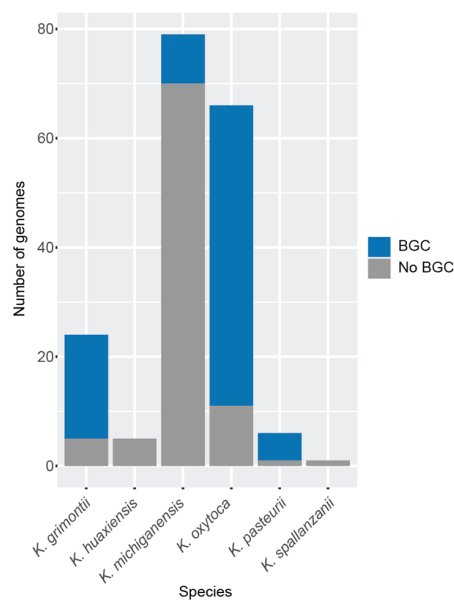

(b)
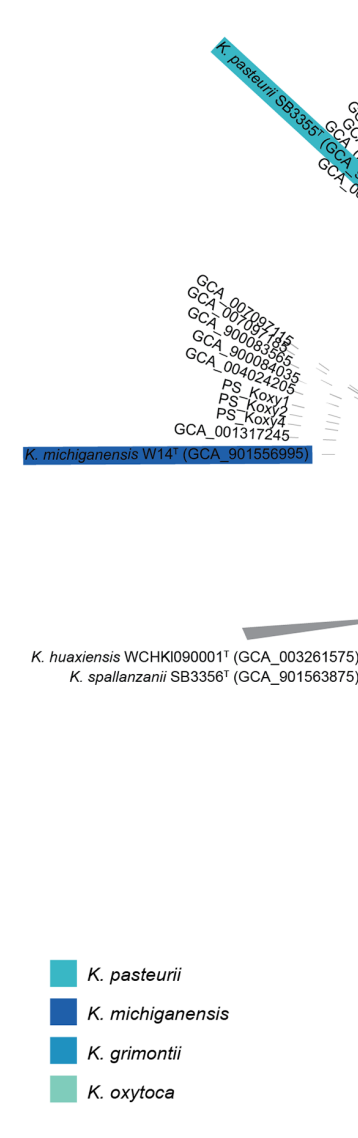

(c)

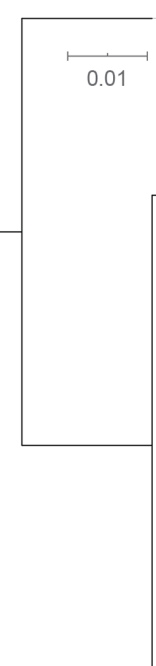

100

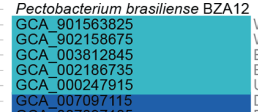

Nound, Paris

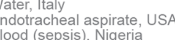

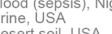

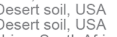

Jrine, South Africa

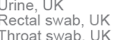

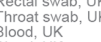

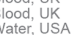

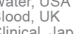

Clinical, Japan
Psammochloa villosa, China

Soil: China

Faeces (preterm infant), UK

Urine, Sweden

Clinical tissue, Swed

(rong

Wound, France
Insect gut, Argentina

Softum, cup, UK
purk

Carrots, Germany

Flood, UK
Baecerm infant), UK Faeces (preterm infant), UK
Ureteral stent, Russia

Blood, UK

Lung, -
Unknown

Cilantro, USA
Blood, UK

Blood, UK
Blood, UK

Blood, UK
Blood, UK

Blood, UK
Urine catheter, USA

Human, USA

Blood (bacteraemia), USA

Urine, USA

Mouse spleen, USA
Mouse intestinal mucosa, USA

Blood, China

Urinary tract, USA
Blood, UK

Blood, UK

Mouse tumour abscess, USA

Blood, UK

aemorrhagic colitis, Canad

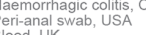

Blood, UK

Blood, UK

lood, U

Paper tray, South Africa Haemorrhagic enterocolitis, Japan

Trachaealaspirate

Blood (bacteraemia), USA
Sink in hospital ward (ICU), USA
Respiratory sample, -

Respiratory sample,
Human, France

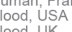

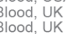

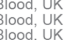

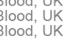

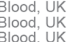

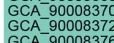

0.1

Blood, UK
Blood, UK
Blood, UK
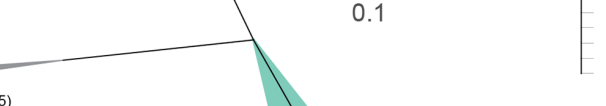

A_900083585

Blood, UK

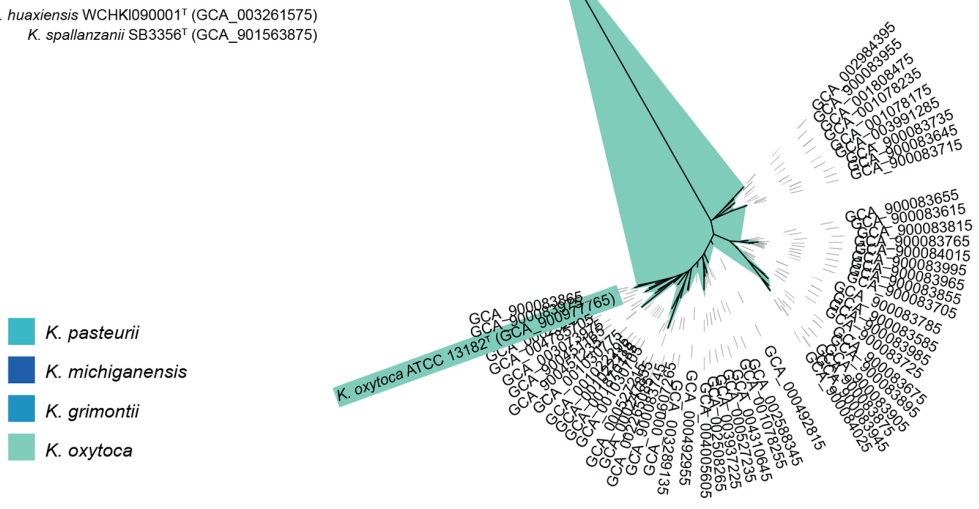

Fig. 3. Distribution of the kleboxymycin BGC in Klebsiella spp. genomes. (a) Distribution of the K. oxytoca complex genomes encoding the entire kleboxymycin BGC. (b) Unrooted maximum-likelihood tree [generated using PhyloPhlAn v0.99 [34] and 380 protein-encoding sequences conserved across the genomes] confirming species affiliations of the 88 genomes within the $K$. oxytoca complex [1] encoding the kleboxymycin BGC. Type strains are shown with coloured backgrounds corresponding to the legend. The clade associated with K. huaxiensis and K. spallanzanii has been collapsed because of space constraints. (c) Maximum-likelihood tree generated with the concatenated protein sequences for the kleboxymycin BGC of the 88 genomes found to encode all 12 genes of the BGC plus the reference sequence [11]. The tree was rooted using the kleboxymycin-encoding BGC of Pectobacterium brasiliense BZA12. Values at nodes, bootstrap values expressed as a percentage of 100 replicates. Sources of isolates, where known, are shown to the right of the assembly accession numbers. (b, c) Scale bar, average number of substitutions per position. 
each gene within the BGC consensus sequences across the four species are available in Table S4.

\section{DISCUSSION}

\section{Genotypic characteristics of the three clinical K. michiganensis strains}

The three clinical strains characterized herein had previously been included in a study of outbreak strains encoding GES-5 and CTX-M-15 [9], the first report of GES-5-positive clinical isolates of K. oxytoca ST138 in the UK. Subsequently, it has been shown that the GES-5 gene in these strains is encoded on an IncQ group plasmid [10]. The whole-genome sequence data reported on previously [9] were not available to us. API 20E (this study; Supplementary Material), MALDI-TOF and limited sequence analysis [9] had shown the strains to be K. oxytoca. Our previous work with isolates recovered from preterm infants had shown that API 20E testing on its own was insufficient to accurately identify K. oxytoca strains [3]. The strains described by Eades et al. [9] were characterized before the availability of MALDI-TOF databases capable of splitting species of the K. oxytoca complex (MALDI-TOF was only able to identify as K. oxytoca but did not have sufficient resolution to identify individual species within the complex) [1]. As we are using PS_Koxy1, PS_Koxy2, PS_Koxy4 in ongoing phage work, we generated draft genome sequences for the strains, to accurately identify them and facilitate detailed host-phage studies in the future.

ANI and phylogenetic analyses confirmed all three strains belonged to the species $K$. michiganensis, not $K$. oxytoca (Supplementary Material). In addition to the AMR genes GES-5 ( $\beta$-lactamase with carbapenemase activity) and CTXM-15 (an ESBL responsible for resistance to cephalosporins) reported previously [9], the strains encoded SHV-66, an ESBL not previously reported in K. oxytoca and related species (Supplementary Material). SHV-66 has previously only been reported in a minority of $\beta$-lactamase-producing $K$. pneumoniae in Guangzhou, China [25]. In this study, SHV-66 (99.65\% identity, bit-score 580 - strict CARD match) was also found in K. michiganensis strains E718 [26], GY84G39 (unpublished), K1439 (unpublished) and 2880STDY5682598 [7] (accession numbers GCA_000276705, GCA_001038305, GCA_002265195 and GCA_900083915, respectively), included in the phylogenetic analysis shown in Fig. S1. Moradigaravand et al. [7] noted in their study that 2880STDY5682598 encoded a $b l a_{\mathrm{SHV}}$ gene, but did not document its type nor indicate its novelty.

The three strains had identical virulence factor profiles (Fig. 3b), encoding the plasminogen activating omptin Pla, the $\mathrm{Mg}^{2+}$ transport proteins MgtBC, Hsp60, autoinducer-2 (LuxS), type I fimbriae, type three fimbriae, type six secretion system I, Escherichia coli common pilus and enterobactin. They also encoded numerous proteins associated with capsule, regulation of capsule synthesis (RcsAB) and LPS, with several of the latter sharing identity with Haemophilus endotoxins (RfaD, GalU, LpxC, GmhA/LpcA, KdsA). All six proteins required for allantoin utilization were encoded in the strains' genomes.

No capsule or $\mathrm{O}$ antigen types could be assigned to the strains using Kaptive, but all three strains were best matched with KL68 [PS_Koxy1, 17 out of 18 genes matched (cpsACP missing); PS_Koxy2 and PS_Koxy4, 16 out of 18 genes matched (cpsACP and KL68_18 missing)] and O1v1 [four out of seven genes ( $w z m, w z t, g l f, w b b O)$ matched in all strains].

\section{MLST sequence types can be used to distinguish members of the $K$. oxytoca complex}

The $b l a_{\text {OXY }}$ gene diversified in parallel to housekeeping genes in the K. oxytoca complex, and it is already known that $r p o B$ - one of the seven genes included in the K. oxytoca MLST scheme [8] - can be used to identify members of the complex [2]. Given that our three clinical strains were ST138 and belonged to K. michiganensis, we determined whether specific sequence types within the MLST scheme could be assigned to species. We found that all species of the $K$. oxytoca complex are associated with specific sequence types. In addition, we identified 10 novel MLST sequence types that can be used to identify K. grimontii, K. michiganensis, K. oxytoca and K. grimontii genomes (Table S2).

Herzog et al. [27], when originally describing the K. oxytoca MLST scheme to characterize clinical isolates, showed their concatenated sequence data for 74 clinical $K$. oxytoca isolates were associated with three clusters (A, B1 and B2). Comparison of their sequence types with our annotations shows that cluster A represents $K$. oxytoca, cluster B1 represents K. michiganensis and cluster B2 represents K. grimontii and K. pasteurii.

The ability to use the K. oxytoca MLST scheme to differentiate clinical isolates will be of particular interest to clinical microbiologists in resource-limited settings who rely on the MLST scheme for typing and epidemiological tracking of isolates in the absence of whole-genome sequence data. It should also be noted that ribosomal MLST [28] (rMLST) available via the Species ID portal of the PubMLST website allows those working with genome sequence data derived from $K$. oxytoca complex isolates to identify species. This resource uses 53 genes encoding the bacterial ribosome protein subunits (rps genes) to rapidly characterize genomic data to the species level.

The identification of ST105 as belonging to Raoultella ornithinolytica indicates this sequence type should be withdrawn from the K. oxytoca MLST scheme.

\section{Distribution of the kleboxymycin BGC in Klebsiella spp.}

As relatively little is known about the virulence factors of $K$. oxytoca and related species, and the VFDB is limited with respect to the number of Klebsiella spp. on which it reports information, we wanted to see whether our strains encoded the kleboxymycin BGC responsible for generating microbiome-associated metabolites known to 
directly contribute to AAHC $[11,12]$. The cytotoxic nature of a heat-stable, non-proteinaceous component of spent media from $K$. oxytoca strains isolated from patients with AAHC was first reported in 1990 [29]. With respect to K. oxytoca being a causative agent of AAHC, the bacterium has fulfilled Koch's postulates [15]. While a commensal of the gut microbiota of some individuals, it has been suggested that cytotoxic $K$. oxytoca is a transient member of the gut microbiota [29].

$\mathrm{TV}$ is a PBD produced by $K$. oxytoca and is a causative agent of AAHC [12]. The TV biosynthesis genes are encoded on a non-ribosomal peptide synthase operon and include $n p s A$, thd $A$ and $n p s B$. The genes aro $X$ and $\operatorname{aro} B$ are also essential for TV production [13]. The genes $n p s A$, thdA, $n p s B$ and aro $X$ are located on a pathogenicity island (PAI). In clinical isolates, the PAI was present in $100 \%$ of toxin-producing isolates, but only $13 \%$ of non-toxin-producing isolates [12]. AAHC is characterized by disruption of epithelial barrier function resulting from apoptosis of epithelial cells lining the colon. TV exerts its apoptotic effect by binding to tubulin and stabilising microtubules, leading to mitotic arrest [14].

A second PBD generated by the same pathway as TV has been identified [13]. TM (also called kleboxymycin [11]) has stronger cytotoxic properties than TV, having a PBD motif with a hydroxyl group at the C11 position, while TV has an indole ring. When deprived of indole by the inactivation of the indole-producing tryptophanase gene tnaA, K. oxytoca produces TM but not TV. TV production is restored with the addition of indole, as indole spontaneously reacts with TM to produce TV. Limited interconversion between TM and TV may also occur spontaneously in vivo [11]. TM is a genotoxin and triggers apoptosis by interacting with DNA, which leads to the activation of damage repair mechanisms, causing DNA strand breakage [14]. DNA interaction is prevented in the case of TV by its indole ring, and both the molecular targets and apoptotic mechanisms of TM and $\mathrm{TV}$ are distinct. The kleboxymycin BGC is not native to K. oxytoca, nor the wider Enterobacteriaceae. Instead, the BGC is thought to have been acquired via horizontal gene transfer from Xenorhabdus spp., which in turn acquired the BGC from bacteria of the phylum Actinobacteria [11].

In the current study, we found the kleboxymycin BGC in our $K$. michiganensis isolates and that it was common among four species of the K. oxytoca complex, with $K$. oxytoca and $K$. grimontii strains making the largest contribution and the type strains of $K$. grimontii and $K$. pasteurii encoding the BGC (Fig. 3). Prior to this study, sequences from two K. oxytoca strains (MH43-1, GenBank accession number MF401554 [11]; AHC-6, GenBank accession number HG425356 [12]) were available for the kleboxymycin BGC. Draft genome sequences do not appear to be publicly available for either of these strains. However, our analysis of the kleboxymycin BGC across the K. oxytoca complex has shown that MH43-1 is a strain of $K$. grimontii (Fig. 3c). Hubbard et al. [20] recently reported on a strain of $K$. grimontii that encoded the BGC, based on antiSMASH analysis. Comparison of the AHC-6 sequence with that of MH43-1 and other sequences included in this study shows AHC-6 is a strain of K. oxytoca $(99.0-99.55 \%$ nucleotide similarity with the BGCs encoded by the three K. oxytoca MAG sequences included in Fig. S5). It is likely that as more genomes of $K$. oxytoca complex species are deposited in public databases, the range of species encoding the kleboxymycin BGC will increase.

All three of our $K$. michiganensis strains encoded the kleboxymycin BGC (Figs 2 and 3), as did strains of $K$. grimontii we previously isolated from preterm infants and $K$. oxytoca and K. michiganensis MAGs recovered from publicly available shotgun metagenomic data (Fig. S5). Whether the BGCs encoded in our clinical and infantassociated strains are functional will be the subject of future studies. The discovery of the kleboxymycin BGC in strains and MAGs recovered from preterm infants is of particular concern. Gut colonization is linked with AAHC, with disease caused by the overgrowth of cytotoxin-producing strains secondary to use of antibiotics [16]. AAHC presents as diffuse mucosal oedema and haemorrhagic erosions [16], and patients pass bloody diarrhoea [30]. The gut microbiota of preterm infants is shaped by the large quantity of antibiotics these infants are given immediately after birth to cover possible early onset infection, with 'blooms' of bacteria preceding onset of infection [3]. Blood in the stool is frequently associated with necrotizing enterocolitis (NEC) in preterm infants, which shares similar pathological hallmarks to AAHC - i.e. intestinal necrosis. Notably, NEC is difficult to diagnose in the early stages and is often associated with sudden serious deterioration in infant health, with treatment options limited due to emerging multi-drugresistant bacteria associated with disease. Previous studies have linked Klebsiella spp. to preterm NEC (supported by corresponding clinical observations), with bacterial overgrowth in the intestine linked to pathological inflammatory cascades, facilitated by a 'leaky' epithelial barrier and LPS-TL4 activation. Recent work has demonstrated K. oxytoca complex isolates of ST173, ST246 and a novel ST [7-32] recovered from infants with NEC can produce kleboxymycin (TM) and TV [31]. Using our MLST annotation scheme (Table S2), we determine these sequence types represent $K$. grimontii, $K$. grimontii and $K$. pasteurii, respectively, with rMLST analyses of the whole-genome sequence data of Paveglio et al. [31] confirming our findings (rST 124484, rST 124487 and rST 157090, respectively). Taken together with the results from our study, we suggest specific virulence factors - i.e. kleboxymycin-related metabolites encoded by atypical Klebsiella spp. - may also play a role in NEC, and this warrants further study.

Attempts have been made to link specific subtypes of K. oxytoca to AAHC [32]. Cytotoxic effects were limited to K. oxytoca, with faecal (and to a lesser extent skin) isolates of $K$. oxytoca most commonly associated with cytotoxicity [32]. No genetic relationship was associated with cytotoxic strains based on pulsed-field gel electrophoresis, and 31 out 
of 97 strains exhibited evidence of cytotoxin production (i.e. reduced viability of Hep2 cells). Joainig et al. [32] isolated genetically distinct cytotoxin-positive and -negative strains from one AAHC patient, leading them to suggest that, when detected in faeces, K. oxytoca should be considered an opportunistic pathogen able to produce disease upon antibiotic treatment. They also found that, in patients with acute or chronic diarrhoeal diseases, more than half of the isolates recovered were cytotoxin-positive. Given that $K$. oxytoca-related species are not routinely screened for in such samples, it is possible that kleboxymycin-producing isolates may make a greater contribution to diarrhoeal diseases than currently recognized, especially in patients suffering from non-C. difficile-associated disease. We have shown that there are species-specific differences in the kleboxymycin BGC (Fig. 3c). These differences may have implications for virulence of strains and warrant further study. It is hoped that the identification of an increased range of strains (including type strains) encoding the kleboxymycin BGC will facilitate such studies.

\section{Funding information}

Imperial Health Charity is thanked for contributing to registration fees for the Professional Doctorate studies of PS. LH was funded by UK Med-Bio (Medical Research Council grant number MR/L01632X/1). The research placement of FM was funded by Nottingham Trent University. LJH is funded by a Wellcome Trust Investigator Award (no. 100/974/C/13/Z); a BBSRC Norwich Research Park Bioscience Doctoral Training grant no. BB/M011216/1 (supervisor LJH, student MK); an Institute Strategic Programme Gut Microbes and Health grant no. BB/R012490/1 and its constituent projects BBS/E/F/000PR10353 and BBS/E/F/000PR10356; and an Institute Strategic Programme Gut Health and Food Safety grant no. BB/J004529/1 to LJH.

\section{Acknowledgements}

Consultant microbiologist Dr Frances Davies (Imperial College Healthcare NHS Trust) is thanked for providing access to clinical strains. This work used the computing resources of CLIMB [33], the UK MEDical BIOinformatics partnership (UK Med-Bio, supported by Medical Research Council grant number MR/L01632X/1) and the Nottingham Trent University Hamilton High Performance Computing Cluster. We would like to thank Dave Baker and the QIB core sequencing team for WGS library preparation and sequencing.

\section{Author contributions}

PS did all phenotypic characterization work. PS did initial bioinformatics analyses (assembly, annotation, CARD, initial BGC work), while FM and $\mathrm{LH}$ undertook the large-scale BGC analyses and infant-associated BGC work; LH undertook the MLST work; ALM and MK prepared and preprocessed DNA for sequencing; LJH, ALM and LH supervised the work. All authors contributed to interpretation and analyses of data, and writing of the manuscript.

\section{Conflicts of interest}

The authors declare that there are no conflicts of interest.

\section{Ethical statement}

The study of anonymized isolates beyond the diagnostic requirement was approved by an NHS research ethics committee (number 06/ Q0406/20).

\section{References}

1. Merla C, Rodrigues C, Passet V, Corbella M, Thorpe HA, et al. Description of Klebsiella spallanzanii sp. nov. and of Klebsiella pasteurii sp. nov. Front Microbiol 2019;10:2360.

2. Passet $\mathrm{V}$, Brisse $\mathrm{S}$. Description of Klebsiella grimontii sp. nov. Int J Syst Evol Microbiol 2018;68:377-381.
3. Chen Y, Brook TC, Soe CZ, O'Neill I, Alcon-Giner C, et al. Preterm infants harbour diverse Klebsiella populations, including atypical species that encode and produce an array of antimicrobial resistance- and virulence-associated factors. Microb Genom 2020:761924.

4. Zheng $B, X u H, Y u X, L v T$, Jiang $X$, et al. Identification and genomic characterization of a KPC-2-, NDM-1- and NDM-5producing Klebsiella michiganensis isolate. J Antimicrob Chemother 2018;73:536-538.

5. Pedersen T, Sekyere JO, Govinden U, Moodley K, Sivertsen A, et al. Spread of plasmid-encoded NDM-1 and GES-5 carbapenemases among extensively drug-resistant and pandrug-resistant clinical Enterobacteriaceae in Durban, South Africa. Antimicrob Agents Chemother 2018:62

6. Seiffert SN, Wüthrich D, Gerth Y, Egli A, Kohler P, et al. First clinical case of kpc-3-producing Klebsiella michiganensis in Europe. New Microbes New Infect 2019;29:100516.

7. Moradigaravand D, Martin V, Peacock SJ, Parkhill J. Population structure of multidrug-resistant Klebsiella oxytoca within hospitals across the United Kingdom and Ireland identifies sharing of virulence and resistance genes with K. pneumoniae. Genome Biol Evol 2017:9:574-584.

8. Jolley KA, Bray JE, Maiden MCJ. Open-access bacterial population genomics: BIGSdb software, the PubMLST.org website and their applications. Wellcome Open Res 2018;3:124.

9. Eades C, Davies F, Donaldson H, Hopkins K, Hill R, et al. GES-5 carpabapenemase-producing Klebsiella oxytoca causing clinical infection in a UK haematopoetic stem cell transplantation unit. 26th European Congress of Clinical Microbiology and Infectious Diseases. Amsterdam, The Netherlands, 2016.

10. Ellington MJ, Davies F, Jauneikaite E, Hopkins KL, Turton JF, et al. A multi-species cluster of GES-5 carbapenemase producing Enterobacterales linked by a geographically disseminated plasmid. Clin Infect Dis Off Publ Infect Dis Soc Am 2019.

11. Tse H, Gu Q, Sze K-. H, Chu IK, RY-T K, et al. A tricyclic pyrrolobenzodiazepine produced by Klebsiella oxytoca is associated with cytotoxicity in antibiotic-associated hemorrhagic colitis. J Biol Chem 2017:292:19503-19520.

12. Schneditz G, Rentner J, Roier S, Pletz J, Herzog KAT, et al. Enterotoxicity of a nonribosomal peptide causes antibiotic-associated colitis. Proc Natl Acad Sci U S A 2014;111:13181-13186.

13. Dornisch E, Pletz J, Glabonjat RA, Martin F, Lembacher-Fadum C et al. Biosynthesis of the Enterotoxic pyrrolobenzodiazepine natural product tilivalline. Angew Chem Int Ed Engl 2017;56:14753-14757.

14. Unterhauser K, Pöltl L, Schneditz G, Kienesberger S, Glabonjat RA, et al. Klebsiella oxytoca enterotoxins tilimycin and tilivalline have distinct host DNA-damaging and microtubule-stabilizing activities. Proc Natl Acad Sci U S A 2019;116:3774-3783.

15. Högenauer C, Langner C, Beubler E, Lippe IT, Schicho R, et al. Klebsiella oxytoca as a causative organism of antibiotic-associated hemorrhagic colitis. N Engl J Med 2006;355:2418-2426.

16. Beaugerie L, Metz M, Barbut F, Bellaiche G, Bouhnik Y, et al. Klebsiella oxytoca as an agent of antibiotic-associated hemorrhagic colitis. Clin Gastroenterol Hepatol Off Clin Pract J Am Gastroenterol Assoc 2003:1:370-376.

17. Seemann T. Prokka: rapid prokaryotic genome annotation. Bioinforma Oxf Engl 2014;30:2068-2069.

18. Jain C, Rodriguez-R LM, Phillippy AM, Konstantinidis KT, Aluru S High throughput ANI analysis of $90 \mathrm{k}$ prokaryotic genomes reveals clear species boundaries. Nat Commun 2018;9:5114.

19. Li H, Handsaker B, Wysoker A, Fennell T, Ruan J, et al. The sequence alignment/map format and SAMtools. Bioinforma Oxf Engl 2009;25:2078-2079.

20. Hubbard ATM, Newire E, Botelho J, Reiné J, Wright E, et al. Isolation of an antimicrobial-resistant, biofilm-forming, Klebsiella grimontii isolate from a reusable water bottle. MicrobiologyOpen 2020;9:1128-1134. 
21. Schliep KP. phangorn: phylogenetic analysis in R. Bioinforma Oxf Engl 2011;27:592-593.

22. Letunic I, Bork P. Interactive Tree Of Life (iTOL) v4: recent updates and new developments. Nucleic Acids Res 2019;47:W256-9.

23. Rodrigues C, Passet V, Rakotondrasoa A, Diallo TA, Criscuolo A, et al. Description of Klebsiella africanensis sp. nov., Klebsiella variicola subsp. tropicalensis subsp. nov. and Klebsiella variicola subsp. variicola subsp. nov. Res Microbiol 2019;170:165-170.

24. Chun J, Oren A, Ventosa A, Christensen H, Arahal DR, et al. Proposed minimal standards for the use of genome data for the taxonomy of prokaryotes. Int J Syst Evol Microbiol 2018;68:461-466.

25. Zuo B, Liu Z-H, Wang H-P, Yang Y-M, Chen J-L, et al. [Genotype of TEM- and SHV-type beta-lactamase producing Klebsiella pneumoniae in Guangzhou area]. Zhonghua Yi Xue Za Zhi 2006;86:2928-2932.

26. Liao T-L, Lin A-C, Chen E, Huang T-W, Liu Y-M, et al. Complete genome sequence of Klebsiella oxytoca E718, a New Delhi metallo- $\beta$-lactamase-1-producing nosocomial strain. J Bacteriol 2012;194:5454.

27. Herzog KAT, Schneditz G, Leitner E, Feierl G, Hoffmann KM, et al. Genotypes of Klebsiella oxytoca isolates from patients with nosocomial pneumonia are distinct from those of isolates from patients with antibiotic-associated hemorrhagic colitis. J Clin Microbiol 2014;52:1607-1616.
28. Jolley KA, Bliss CM, Bennett JS, Bratcher HB, Brehony C, et al. Ribosomal multilocus sequence typing: universal characterization of bacteria from domain to strain. Microbiol Read Engl 2012;158:1005-1015.

29. Higaki M, Chida T, Takano H, Nakaya R. Cytotoxic component(s) of Klebsiella oxytoca on HEp-2 cells. Microbiol Immunol 1990;34:147-151.

30. Youn Y, Lee SW, Cho H-H, Park S, Chung H-S, et al. Antibioticsassociated hemorrhagic colitis caused by Klebsiella oxytoca: two case reports. Pediatr Gastroenterol Hepatol Nutr 2018;21:141-146.

31. Paveglio S, Ledala N, Rezaul K, Lin Q, Zhou Y, et al. Cytotoxinproducing Klebsiella oxytoca in the preterm gut and its association with necrotizing enterocolitis. Emerg Microbes Infect 2020;9:1321-1329.

32. Joainig MM, Gorkiewicz G, Leitner E, Weberhofer P, Zollner-Schwetz I, et al. Cytotoxic effects of Klebsiella oxytoca strains isolated from patients with antibiotic-associated hemorrhagic colitis or other diseases caused by infections and from healthy subjects. J Clin Microbiol 2010;48:817-824.

33. Connor TR, Loman NJ, Thompson S, Smith A, Southgate J, et al. CLIMB (the Cloud Infrastructure for Microbial Bioinformatics): an online resource for the medical microbiology community. Microb Genomics 2016;2:e000086.

34. Segata N, Börnigen D, Morgan XC, Huttenhower C. PhyloPhlAn is a new method for improved phylogenetic and taxonomic placement of microbes. Nat Commun 2013;4:2304.

\section{Five reasons to publish your next article with a Microbiology Society journal}

1. The Microbiology Society is a not-for-profit organization.

2. We offer fast and rigorous peer review - average time to first decision is 4-6 weeks.

3. Our journals have a global readership with subscriptions held in research institutions around the world.

4. $80 \%$ of our authors rate our submission process as 'excellent' or 'very good'.

5. Your article will be published on an interactive journal platform with advanced metrics.

Find out more and submit your article at microbiologyresearch.org. 